\title{
Inteligibilidad de la Docencia de Pregrado: de la Enseñanza por Objetivos al Desarrollo de Competencias
}

Miguel A. Bustamante ${ }^{(1)(*)}$, Maderline L. Grandón(2), M. del Carmen Lapo ${ }^{(3)}$ y Claudio H. Oyarzún ${ }^{(4)}$

(1) Escuela de Ingeniería Comercial, Facultad de Economía y Negocios, Universidad de Talca, Chile.Campus Lircay. Avenida Lircay S/N, Talca-Chile (e-mail: mabu@utalca.cl)

(2) Centro de Gestión en Instituciones de Salud, CEGIS, Universidad de Talca, Chile.Campus Lircay. Avenida Lircay S/N, Talca-Chile (e-mail: mgrandon@utalca.cl)

(3) Facultad de Ciencias Económicas y Administrativas de la Universidad Católica de Santiago de Guayaquil, Ecuador (e-mail: maria.lapo@cu.ucsg.edu.ec)

(4) Instituto de Investigación y Desarrollo Educacional, IIDE, Universidad de Talca, Chile. Campus Lircay. Avenida Lircay S/N, Talca-Chile (e-mail: cloyarzun@utalca.cl)

* Autor a quien debe ser dirigida la correspondencia

Recibido Ene. 8, 2016; Aceptado Mar. 7, 2016; Versión final Abr. 6, 2016, Publicado Oct. 2016

\section{Resumen}

El presente trabajo determina los elementos que definen la inteligibilidad de la práctica docente de pregrado de dos Universidades públicas chilenas que transitan desde la enseñanza tradicional por objetivos hacia la enseñanza con enfoque en competencias. El estudio es cualitativo, no experimental y exploratorio y se realizó mediante el análisis de las actividades académicas relatadas por los propios profesores de diversos perfiles y especialidades. Los datos fueron procesados mediante el análisis de contenido de los discursos escritos recogidos de una muestra de 66 profesores. Se concluye que existen prioridades divergentes entre los docentes de la Universidad "A", con énfasis en las competencias, respecto de los docentes de la Universidad "B" que se enfocan en la enseñanza por objetivos. En general, los principios orientadores de los académicos se basan principalmente en la enseñanza tradicional por objetivos. Sin embargo, la enseñanza basada en competencias está siendo incorporada de manera progresiva a la práctica docente habitual.

\section{Intelligibility of Undergraduate Teaching: From Teaching for Objectives to Development of Competitions}

\begin{abstract}
This paper determines elements that define the intelligibility of undergraduate teaching practice in two Chilean public universities which are in the process of changing from traditional teaching by objectives to a competency-based education. The study is qualitative, not experimental and exploratory and was performed by analyzing academic activities reported by teachers of different profiles and specialties. The data were processed using analysis of content of written speeches collected from a sample of 66 teachers. It is concluded that there are divergent priorities among teachers of the University "A", with powerful emphasis on competences and teachers of the University "B" that focus on teaching by objectives. In general, the guiding principles of professors were based mainly in the traditional teaching objectives, however gradually; competency-based education is being incorporated into the usual teaching practice.
\end{abstract}

Keywords: teaching and training; professional training; pedagogical practice; teaching method; competences 


\section{INTRODUCCIÓN}

La educación con enfoque en competencias apela al constructivismo y a la reflexión de la práctica en aula asignando un rol activo a las personas que aprenden (Organización para la Cooperación y el Desarrollo Económico [OCDE], 2004). Asume que el estudiante concurre con sus percepciones, significaciones y sentido de realidad y se integra a un proceso gradual y singular que conecta conceptos y preguntas en un ciclo interactivo permanente de enseñanza - aprendizaje (Crisol, 2010; Vadillo y Klinger, 2004; Perrenoud, 2001). Por una parte, el aprendizaje es en sí mismo una competencia que simultáneamente exige saber movilizar capacidades, estrategias y destrezas en una totalidad coherente $y$, por otra, el método faculta a la persona a adquirir y utilizar conocimientos declarativos y procedimentales dando forma a un saber-hacer singular y complejo denominado competencias (De Miguel, 2005; Ley Orgánica Española [LOE], 2007).

En el contexto internacional y recogiendo experiencias de innovación curricular, el Tecnológico Nacional de México, ha implementado un modelo curricular utilizando un proyecto formativo integrado, sustentado en las concepciones de formación profesional, aprendizaje y práctica educativa. La perspectiva de esta entidad, para dar sentido y significado a los contenidos educativos, al quehacer del profesorado y del estudiantado, así como a las estrategias de aprendizaje y didácticas, ha sido el diseño, implementación, desarrollo, seguimiento y evaluación de los programas académicos. Entre sus logros se mencionan mayor flexibilidad, integración del saber y del saber hacer, propiciando el aprendizaje continuo de estudiantes y el desarrollo de competencias profesionales (Acosta y Gamino, 2016).

También como una innovación, se menciona la identificación de competencias administrativas y académicas en el profesorado de educación media superior, realizado en Ciudad Juares, Chihuahua, México que, ejecutado sobre la base de metodologías cualitativas, logró identificar el aprendizaje en competencias administrativas relevantes en la planificación, con un diseño instrumental evaluativo y de gestión institucional. Entre sus resultados se identifican algunas preocupaciones en torno a la formación y ejecución de competencias académicas en los procesos pedagógicos y ante la evaluación docente que una innovación de esta naturaleza implica (Castro, 2015).

Para algunos, el docente es un facilitador que ordena y estructura el aprendizaje para que el alumno elabore su conocimiento, además, guía al estudiante en la resolución de sus necesidades de formación configurando un rol que supera el modelo de transmisión a memoria o de enseñanza por objetivos que se define como el prototipo de las enseñanza tradicional (Marín-García y Conchado-Peiró, 2012; Camargo y Pardo, 2008; Cerrillo e Izuzquiza, 2005; Batanaz, 1998). El tutor por su parte, desde la perspectiva de la función tutorial (Barbier, 1999a), es consustancial al rol del docente en la enseñanza con enfoque en competencias (Camargo y Pardo, 2008) en tanto estimula la conciencia de trabajo y la reflexión sistemática del que aprende y colabora en "la descripción, interpretación y búsqueda de conexiones a partir de eventos significativos..." (Patiño y Rojas, 2009) e introduce cambios metodológicos fundamentales en el modo en que se enseña y en cómo, el profesor, se concibe en el proceso de enseñanza (Palominos et al., 2014; Díaz et al., 2010; Barbier y Galatanu, 2004). En consecuencia, el docente - tutor ha de enfrentar contextos socio-educativos diversos y complejidades derivadas de sus propias convergencias - divergencias y creencias en torno al denominado ciclo enseñanza-aprendizaje (Díaz et al., 2010; García et al., 2008) que, terminan por caracterizar el dinamismo y necesidad de autorregulación oportuna del rol del tutor, de las propias actuaciones y de los conocimientos que transmite respecto de las percepciones de claridad, consistencia, calidad y criterio de evaluación del aprendiz (Coronado y Estupiñán, 2012; Patiño y Rojas, 2009).

Por otra parte, analizadas diversas experiencias de implementación de métodos de formación de profesionales (Ortiz y Cires, 2012), se ha podido ratificar la importancia y necesidad de adoptar el enfoque de competencias y la preparación de diseñadores y ejecutores del proceso, así como también que es necesario estudiar el valor metodológico de las innovaciones para la formación de los futuros profesionales con enfoque en competencias (Legault, 2012), así como también desarrollar competencias específicas de los docentes (Carrera y Cioduras, 2012) incorporando la cultura del cambio y el mejoramiento continuo (Pavié, 2011; Moreno, 2010). Ahora, desde la perspectiva de las preocupaciones sobre propuestas curriculares y formativas se ha llegado a concluir que, "mientras una determinada concepción de las competencias y de su modo de implementarlas puede incrementar el grado de relevancia de la formación universitaria, otra forma de concebirlas y de entender el proceso de su desarrollo en los nuevos planes de formación, puede acabar, cuando menos, en un clamoroso ejercicio de irrelevancia" (Rué, 2008), asunto que ha de ser previsto si el propósito de cambio en el enfoque de enseñanza quiere ser efectivamente consolidado. 
A nivel europeo, el denominado enfoque de competencias - CBET, Competencies Based Education and Training- muestra un relativo éxito en algunos países anglosajones, sin embargo, su introducción en España ha sido difícil, por la formulación de títulos en forma de competencias, así como por las dificultades de comprensión del enfoque por parte de las Universidades (Carabaña, 2011). Por otra parte, el efecto, a veces no previsto de estas innovaciones, impacta la formación inicial de los docentes cuyo beneficio es que el desarrollo de competencias se vincula con la adecuada inserción de los profesionales en los equipos de trabajo (Labra et al., 2011).

Desde la perspectiva del análisis de prácticas y actividades, el rol docente se focaliza en la doble reflexión de la acción y del saber que resulta esencial para la generación de conocimiento nuevo (Bustamante et al., 2009). El conocimiento, en su calidad de constructo complejo, se basa en parte, en el conocimiento prescrito (desarrollado y enseñado) y del que deriva de la experiencia acumulada de la práctica y de un proceso de generación progresiva en continuidad (Dewey, 1926). De ahí entonces que experiencia, acción y resultados, deben pasar por la reflexión como elemento intelectual que contribuye al aprendizaje (Greca y Moreira, 1997) y es, la reflexión, la que permite que una experiencia termine siendo educativa cuando la persona se involucra y está dispuesta a cambiar el sentido de sus experiencias a futuro (Popper, 1994).

Sobre la base del rol del docente-tutor, del análisis de la práctica docente y de la experiencia de quien aprende, el presente trabajo estudia los mecanismos, rutinas y prácticas de enseñanza en pregrado, para generar la reflexión e inteligibilidad del quehacer docente (Moreno et al., 2014; Barbier, 1999b) y, en consecuencia, determinar cómo es que el docente asume su propia función y de qué forma reconoce la virtualidad y vitalidad de su quehacer a través del análisis como actor principal "... al cual se debe apuntar para lograr cambio e innovaciones en la educación" (Rodríguez, 2000).

Los estudios de inteligibilidad se orientan a la toma de conciencia de los espacios de opacidad del desempeño profesional y hace factible el desarrollo de competencias complejas y la construcción de nuevos modelos mentales (Johnson-Laird, 1990) en torno a la profesionalización de las actividades, para generar aprendizaje de la "experiencia" y "auto formación" en "singularidad" de quien aprende (Barbier, 2000). En consecuencia, la inteligibilidad dice relación con lo que los profesionales "deben hacer" o bien con lo que éstos "hacen realmente" y de lo cual logran comprensión mediante el "análisis de prácticas", como método de toma de razón de las actividades realizadas, del "trabajo prescrito" o del "trabajo real" ejecutado en un cierto contexto determinado por la organización, la unidad o los dominios profesionales que le dan validez (Barbier, 1999b; 2000; 2001).

Por último, en el desarrollo de la inteligibilidad se recorren a lo menos cinco pasos. Primero, que los practicantes expertos tienen más conocimientos del que ellos mismos puedan percatarse, decir o expresar; segundo, que dichos expertos no siguen modelos prescritos; tercero, que los expertos utilizan la reflexión implícita en la acción para adaptar sus prácticas a nuevas situaciones; cuarto, que la reflexión tiene límites y se apoya en la investigación y la experimentación y finalmente, en quinto lugar, que el experto se da cuenta que se puede educar a sí mismo y a nuevos practicantes mediante la práctica reflexiva y la toma de conciencia de sus desempeños e implicaciones. A esto se denomina inteligibilidad (Barbier, 1999b; 2000; 2001).

El objetivo del presente trabajo es entonces, determinar los elementos que definen la inteligibilidad de la práctica docente de pregrado de dos Universidades públicas chilenas denominadas "A" y "B" que transitan desde la enseñanza tradicional por objetivos hacia la enseñanza con enfoque en competencias.

\section{METODOLOGÍA}

El estudio se desarrolló utilizando el método de entrevista de explicitación del análisis de actividades (Barbier, 1996a; Barbier y Galatanu, 2000). Para ello se solicitó a los profesores un relato abierto y espontáneo de sus actividades docentes (Barbier, 2004). El pedido explícito fue de un relato de la práctica docente, tal y como fuera entendido y expresado por los entrevistados, sin mayor orientación terminológica para evitar sobreentendidos que produjeran un efecto sesgo. Los registros recogidos, se estructuraron en un sistema de ítems o palabras fuerza, derivados de los propios relatos, que permitieron decodificar y dar sentido interpretable a las actividades relatadas (Barbier, 2000; Barbier et al., 2006). De esta forma, se recogieron las reflexiones del desempeño docente en su contexto organizacional, de la unidad y comprensión personal de la actividad realizada, como fuente de registro y aproximación no experimental ex post del fenómeno estudiado (Barbier, 1985).

Es sabido, además, que el individuo ubicado dentro de un determinado contexto pone en práctica sus talentos y se ajusta a un esquema de trabajo y a regulaciones que orientan sus decisiones, su forma de hacer y su implementación. Sin embargo, es natural que las actividades se hagan evidentes a quienes las 
observan tomando la forma de acciones, huellas e impactos que afectan de múltiples formas sus propios contextos de desempeño. Acciones que se ejecutan en un "tiempo institucional" determinado, se articulan en interacción con un cierto "espacio profesional" pero que devienen de un determinado "espacio privado" que el individuo pone en movimiento primero, en un "espacio organizacional" y seguidamente en un "espacio industrial" determinado (Spencer, 1993; Johnson-Laird, 1990).

El procesamiento se realizó mediante la técnica del análisis de contenido (López, 2002) identificando elementos representativos tales como palabras y frases que fueron clasificadas como variables y categorías para explicitar el fenómeno indagado (Abela,2002) y caracterizar percepciones, sentimientos $y$ concepciones de los profesores de pregrado frente a su desempeño docente en un contexto de cambio e innovación curricular, desde la enseñanza tradicional hacia la enseñanza basada en competencias, y en sus respectivas universidades.

El análisis de registros permitió una caracterización general, referida a una interpretación generalizada que se hace de los datos como una primera aproximación a la comprensión de las actividades (Barbier, 2000; Barbier et al., 2005), por medio de la mayor o menor frecuencia de las palabras más recurrentes en los relatos, de las actividades descritas por los docentes.

El denominado análisis por categorías, facilitó una interpretación más exhaustiva de las palabras (Barbier, 1996b; 1985) incluyendo tanto las más como las menos frecuentes para generar una tabla de frecuencias estandarizadas respecto de la media de términos que se ubican a la izquierda (con signo negativo) y a la derecha (con signo positivo) de la curva normal.

Por último, los principios, categorías y cobertura dicen relación, por su parte, con las combinaciones de palabras significativas y constructos semánticos que explicitan conceptos más complejos dentro de los relatos analizados (Argyris y Schön, 1992; Barbier, 19990a; 2001) y corresponden también a descripciones recurrentes sobre las cuales se calcularon frecuencias relativas que se denominaron cobertura, es decir, el grado de generalización porcentual de las palabras más significativas recogidas dentro de los relatos.

Se utilizó el muestreo no probabilístico por conveniencia seleccionando profesores de pregrado inmersos en su realidad y que hubiesen expresado su voluntad de contribuir al estudio (Fernández, 2002). Se obtuvo información de 66 docentes de pregrado de los cuales 32 corresponden a la Universidad "A" y 34 a la Universidad "B". Los elementos de la muestra son profesores de las plantas académicas permanentes de cada una de las Universidades y provenientes de diversas especialidades profesionales.

La Universidad "A", es la que lleva más años en Chile aplicando esta metodología de enseñanza, puesto que, desde el año 2003, ha incorporado entre sus características estratégicas distintivas de enseñanza, la formación con base en competencias y que se expresa en su plan estratégico institucional y en el proyecto educativo como factor distintivo y diferenciador. La Universidad "B" por su parte, se encuentra desde hace a lo menos 5 años en un plan sistemático de inducción de sus profesores en la enseñanza con enfoque en competencias, reflejado en su plan estratégico institucional y en la implementación de programas formales de formación de sus académicos en dicha innovación metodológica.

El análisis de datos se efectuó a través del software Nvivo 10, determinándose frecuencias absolutas y estandarizadas de palabras y frases significativas que dan forma a categorías semánticas que dan sentido y significación al fenómeno estudiado (Checchia, 2009). Mediante la fórmula (1), se determinó un listado de palabras recurrentes que constituyen sistemas de categorías identificables, donde $X_{i}$ corresponde al valor de la variable $i$, y $n$ al número de variables.

$$
\sum X i / n
$$

En tanto que, para comparar dichas expresiones con las restantes dentro del sistema en análisis, se procedió a la estandarización de las frecuencias en torno a la media descontando el efecto de la dispersión de los datos a través de la fórmula (2), Donde $X$ es el valor de la variable estandarizada, $X_{i}$ corresponde al valor de la variable $i, \mu$ representa el promedio de las variables $i$ analizadas, y $\sigma$ corresponde a la desviación estándar de los promedios de las variables $i$.

$$
X=(X i-\mu) / \sigma
$$

En ésta, los resultados se ubican entre los valores $(-3)$ y $(+3)$, siendo $(0)$ el valor central que se corresponde con el promedio. 


\section{RESULTADOS}

Los resultados del estudio se presentan, primero con una caracterización general, seguido de un análisis focalizado por Universidad para terminar con una caracterización de principios, categorías y cobertura.

\section{Caracterización general}

En general, los docentes de la Universidad "A" (Tabla 1) enfocan su docencia hacia las actividades, aprendizaje y experiencias con 2,91, 1,72 y 1,03 puntos por encima de la media estándar. En su opuesto y con -0,81 puntos estándar se identifican las palabras buscar, gestión, apoyar e implementación ratificando que su actividad es crear $(-0,90)$ espacios para el aprendizaje de sus estudiantes. En los relatos se habla de un aprendizaje implícito capaz de develar el hecho de que la actividad docente no sólo es de transmisión sino que implica un rol activo de tutoría a partir de prácticas de retroinformación eficaces.

A nivel agregado en la Universidad "B" (Tabla 1), se dieron las palabras buscar y contexto con 2,06 puntos permitiendo inferir que los académicos enfocan su enseñanza en la indagación para la solución de problemas y donde el término contexto refiere a los condicionamientos geográficos y de aislamiento que los estudiantes enfrentan. A estas expresiones le sigue la palabra actividades con 1,82 puntos, que define el énfasis que los docentes le asignan a un sistema de prácticas, actitudes y conductas para el desarrollo de competencias.

Complementariamente, con frecuencia estándar de $-0,83$, se identificaron las palabras competencias, experiencias, formación/formar, responsabilidad, enseñar, gestión, apoyar e implementación, todas relacionadas en forma directa con el modelo de formación basado en competencias pero que, aunque ha sido el asumido institucionalmente por la Universidad, aún no está en régimen dentro de la práctica docente habitual de sus profesores.

Tabla 1: Frecuencia Estandarizada $(\sigma)$ Global por categoría de palabras entre rangos \pm 3

\begin{tabular}{|l|c|c|}
\hline Palabras & $\sigma$ Universidad "A" 2014 & $\sigma$ Universidad "B" 2013 \\
\hline Actividades & 2,91 & 1,82 \\
\hline Aprendizaje & 1,72 & 0,37 \\
\hline Desarrollar & $-0,04$ & 0,62 \\
\hline Conocimiento & 0,31 & 0,13 \\
\hline Contenidos & 1,07 & $-0,59$ \\
\hline Competencias & $-0,49$ & $-0,83$ \\
\hline Experiencias & 1,03 & $-0,83$ \\
\hline Manera & 0,09 & 0,62 \\
\hline Contexto & 0,09 & 2,06 \\
\hline Formación/formar & 0,37 & $-0,83$ \\
\hline Evaluación & $-0,45$ & $-0,35$ \\
\hline Responsabilidad & $-0,41$ & $-0,83$ \\
\hline Enseñar & $-0,49$ & $-0,83$ \\
\hline Buscar & $-0,81$ & 2,06 \\
\hline Metodologías/método & $-0,65$ & $-0,35$ \\
\hline Docencia & $-0,73$ & 0,37 \\
\hline Gestión & $-0,81$ & $-0,83$ \\
\hline Apoyar & $-0,81$ & $-0,83$ \\
\hline Implementación & $-0,81$ & $-0,83$ \\
\hline Crear & $-0,90$ & $-0,11$ \\
\hline
\end{tabular}

Análisis de categorías

Las categorías de palabras más significativas que los docentes de la Universidad "A" sitúan en alta jerarquía son aprendizaje con 2,39 puntos, desarrollo, objetivos y contenidos que alcanzan 1,34 puntos y experiencias con 1,28 puntos estandarizados, acercando progresivamente la práctica docente hacia el modelo con enfoque en competencias. 
Entre los términos con menor frecuencia se menciona docencia con -1,15, educación y trabajar que alcanza -1,01 puntos y tarea con -1,08 a la izquierda de la media estandarizada, explicitando cómo el logro de metas y los objetivos son el resultado de la formación tradicional basada en objetivos de enseñanza (Tabla 2). Por otra parte, los docentes de la Universidad "B" presentan un perfil enfocado principalmente en lo geográfico territorial a través de los términos contexto y búsqueda con 1,84 puntos seguidas de actividades con 1,57 e información con 1,02 puntos estándar. En tanto que, con menores frecuencias mencionan las palabras evaluación con -1,15 puntos seguida de metodología, prácticas, preparación, trabajo, contenidos/materia con - 0,88 puntos estándar por debajo de la media, describiendo cómo sus actividades de pregrado giran aún alrededor de conductas tradicionales de enseñanza por objetivos (Tabla 2).

Tabla 2: Frecuencia estandarizada $(\sigma)$ global por categoría de palabras entre rangos \pm 3

\begin{tabular}{|l|c|c|c|c|c|c|c|}
\hline \multicolumn{4}{|c|}{ Universidad "A" 2014 } & \multicolumn{4}{c|}{ Universidad "B" 2013 } \\
\hline Palabras & $\sigma$ & Palabras & $\sigma$ & Palabras & $\sigma$ & Palabras & $\sigma$ \\
\hline Aprendizaje & 2,39 & Capacitación & $-0,36$ & Contexto & 1,84 & Interactuar & $-0,34$ \\
\hline Desarrollo & 1,34 & Desarrollar & $-0,36$ & Búsqueda & 1,84 & Manera & $-0,61$ \\
\hline Objetivos & 1,34 & Logro & $-0,43$ & Actividades & 1,57 & Metodología & $-0,88$ \\
\hline Contenidos & 1,34 & Responsabilidad & $-0,49$ & Información & 1,02 & Prácticas & $-0,88$ \\
\hline Experiencias & 1,28 & Desempeño & $-0,69$ & Desarrollar & 0,21 & Preparación & $-0,88$ \\
\hline Formación & 0,23 & Educación & $-1,01$ & Docencia & 0,21 & Trabajo & $-0,88$ \\
\hline Manera & $-0,10$ & Trabajar & $-1,01$ & Aprendizajes & $-0,06$ & Contenidos/Materia & $-0,88$ \\
\hline Conocimientos & $-0,16$ & Tarea & $-1,08$ & Conocimientos & $-0,06$ & Evaluación & $-1,15$ \\
\hline Contexto & $-0,23$ & Docencia & $-1,15$ & Crear & $-0,06$ & & \\
\hline Grupo & $-0,23$ & & & & & & \\
\hline
\end{tabular}

\section{Principios, categorías y cobertura}

En general, para los profesores de la Universidad "A" (Tabla 3), el principio orientador más recurrente fue aprendizajes significativos con 1,08 puntos, en tanto que el menos referenciado fue actividad y participación con el -1.08 puntos a la izquierda de la normal estándar. Por su parte, para los profesores de la Universidad "B", el principio orientador más frecuente fue globalización con 1,08 puntos estándar y el menos referenciado resultó motivación y autoestima con -1,62 puntos.

Del análisis de frecuencias por categorías de palabras, se puede afirmar que los docentes de la Universidad "A" presentan los porcentajes más altos de cobertura en torno a la actividad de guiar con un $52,21 \%$ que se expresa en acciones de aprendizaje, desarrollo, objetivos y contenidos a las cuales se suma experiencias, cuando fijan objetivos sin descuidar el contexto situacional en el cual se encuentran y donde su acción de tutoría reconoce al estudiante como agente activo de su propio aprendizaje interactuando tanto a nivel alumno-alumnos como a nivel alumnos-profesor. Además, los profesores mencionan también la categoría diagnóstico con un 35,65\% de cobertura, al abrirse a nuevas prácticas requeridas por el método de enseñanza con enfoque en competencias como es el hecho de enseñar a través de la evidencia y sobre la base de la personalización y adaptación de los métodos a la diversidad de capacidades de aprendizaje de sus estudiantes.

Finalmente, la categoría aportar alcanzó un significativo 31,26\% de cobertura en referencia a la didáctica de trabajo en equipo y las actividades de retroalimentación en congruencia con el principio de capacitar citado en la fase pasos de la formación por competencias. Dentro de las categorías con menos frecuencias, se menciona articular y educar que básicamente se ejecutan con relación a los dominios cognitivos de la enseñanza por objetivos. Los docentes de la Universidad "B" (Tabla 3), enfatizan la categoría guiar con $51,51 \%$ de cobertura determinada en los principios de actividad y participación, aprendizajes significativos, globalización, y personalización, infiriéndose que los alumnos son guiados en un proceso de enseñanza aprendizaje constructivista eventualmente, para hacer realidad el lema central de la universidad de guiarlos en su desarrollo como futuros profesionales. Le sigue en frecuencia la actividad de planificar con 59,61\% de cobertura según la cual el docente prepara su docencia asumiendo un alumno promedio o superior puesto que, lo que interesa, es que los estudiantes se hagan parte de un proceso social de aprendizaje colectivo, donde cada quien aporta lo suyo a la construcción compartida de un aprendizaje complejo. En lo formativo, las prácticas de enseñanza incorporan la actividad pasos con un 34,63\% de cobertura, mostrando que los docentes también realizan actividades de entrenamiento con propósitos de capacitación y desarrollo de destrezas, lo cual implica que los estudiantes deben ejercitarse y reconocer condicionamientos de un entorno agresivo pero que, al mismo tiempo, les hace sujetos singulares capaces de internalizar destrezas profesionales complejas. 
Por último, la actividad evaluar con $11,32 \%$ de cobertura es una de las menos referenciadas en la práctica docente y parece implementada bajo métodos didácticos tradicionales. Sin embargo, a partir del método de formación basada en competencias, los alumnos son parte activa de sus propias evaluaciones y, en ocasiones, partícipe de la evaluación de sus compañeros a través de co-evaluaciones y retroalimentaciones mutuas. De este modo se referencia el evaluar por pares con un 4,62\% de cobertura que muestra cómo los docentes de la Universidad "B" incorporan, progresivamente, nuevos procedimientos de evaluación a su práctica docente tradicional.

Tabla 3: Principios orientadores y cobertura de palabras a nivel de pregrado

\begin{tabular}{|c|c|c|c|}
\hline \multirow{2}{*}{\multicolumn{2}{|c|}{ Principios/ Categorías }} & \multicolumn{2}{|c|}{ Principios orientadores Valores estandarizados $(\sigma)$} \\
\hline & & Universidad "A" 2012 & Universidad "B" 2011 \\
\hline \multicolumn{2}{|l|}{ Actividad Y Participación } & $-1,08$ & 0,0 \\
\hline \multicolumn{2}{|l|}{ Motivación Y Autoestima } & 0,54 & $-1,62$ \\
\hline \multicolumn{2}{|l|}{ Aprendizajes Significativos } & 1,08 & $-1,08$ \\
\hline \multicolumn{2}{|l|}{ Globalización } & 0,00 & 1,08 \\
\hline \multicolumn{2}{|l|}{ Personalización } & $-0,54$ & 0,00 \\
\hline \multicolumn{2}{|l|}{ Interacción } & 0,54 & $-1,62$ \\
\hline \multicolumn{4}{|c|}{ Cobertura de palabras pregrado $2013-2014$} \\
\hline Universidad "A" 2014 & $\%$ & Univers & $\%$ \\
\hline Aportar & 31,26 & & 9,59 \\
\hline Aprender & 9,94 & & 4,67 \\
\hline Guiar & 52,21 & & 51,51 \\
\hline Evaluar & 20,33 & & 11,32 \\
\hline Educar & 18,42 & & 19,39 \\
\hline Ensañar & 29,55 & & \\
\hline Planificar & 17,48 & & 59,61 \\
\hline Estructurar & 28,34 & & 27,1 \\
\hline Capacitar & 16,78 & & 10,51 \\
\hline Diagnóstico & 35,65 & & 19,57 \\
\hline Motivación & 25,72 & & 5,3 \\
\hline Proceso & 15,23 & & 34,63 \\
\hline Conceptos & 16,8 & Evalua & 4,62 \\
\hline Articular & 9,91 & & 18,21 \\
\hline Correcciones & 17,96 & Progr & 20,67 \\
\hline Autorrealización & 10,36 & & \\
\hline Objetivos & 15,68 & & \\
\hline Contenidos & 20,11 & & \\
\hline
\end{tabular}

Los docentes de la Universidad "A" ponen el énfasis sobre los objetivos pedagógicos que deben cumplir, les es imprescindible valorar una competencia de forma integral, al mismo tiempo que se evalúa el conocimiento, la comprensión, la solución de problemas, los niveles técnicos, las actitudes y principios éticos en la evaluación, confirmando el hecho de que los estudiantes deben desarrollar habilidades y que ellos las deben propiciar apoyados en prácticas de enseñanza-aprendizaje sobre la base de las categorías motivación y autoestima, aprendizajes significativos, e interacción. De este modo en ésta casa de estudios superiores se entrega a todos sus estudiantes una formación basada en competencias, donde claramente destaca el hecho de que los docentes deben ser los soportes de las nuevas prácticas de enseñanzaaprendizaje, situación que no es ajena a docentes que asumen esto como un verdadero desafío. Los docentes de la Universidad "B" validan como determinante el contexto en el cual se encuentran, circunscritos a una ciudad, al clima propio de su zona y la cultura, entre otros, y la necesidad de lidiar con dichos factores. Los profesores, muestran resiliencia individual y coherencia con las prácticas resultantes en relación con el objetivo de aminorar el impacto del medio que se hace evidente en el intento de adecuarse a su realidad. 
Se determina de esta forma un perfil del docente de pregrado centrado en generar las condiciones necesarias para llevar a cabo su desempeño, y enfrentar de la mejor manera posible las condicionantes del medio, en un proceso desafiante y motivador para sus estudiantes. Es por ello que su práctica tiende a estar mayormente en sintonía con la búsqueda de metodologías adecuadas a su realidad, a establecer una planeación concienzuda de las actividades a realizar, así como a prever el modo en que éstas se llevarán a cabo desarrollando métodos asertivos de enseñanza y evaluación que pone especial atención en la estructuración del trabajo en aula, en la que el docente desarrolla actividades basadas principalmente en los principios de "Globalización, Actividad - Participación, y Personalización".

El presente estudio contribuye de manera significativa a la comprensión de la actividad educativa, define el rol del académico como actor social relevante en la formación de personas y profesionales donde "la profesionalidad del profesor universitario se basa en la reflexión sobre su propia tarea" que se hace evidente a través del relato único y subjetivo de académicos que, sin perjuicio de provenir de profesiones y contextos diferentes, logran interesantes coincidencias en relación con modos, formas, estrategias y técnicas de enseñanza.

\section{CONCLUSIONES}

1. En general, se observan grados de inteligibilidad divergentes entre los docentes de las Universidades analizadas. Las palabras más frecuentes tales como "actividades, aprendizaje y experiencias" con promedios estandarizados altos, a la derecha de la normal, en una escala de 3 puntos, caracterizan a los docentes de la Universidad "A", como aquella con más énfasis competencial, respecto de los docentes de la Universidad "B" que focalizan la enseñanza por objetivos y que se manifiestan en palabras tales como "buscar y contexto" también con frecuencias estandarizadas altas, y que se ubican en la parte positiva de la curva normal. En resumen, los profesores de ambas universidades, aunque con lógicas docentes diferenciadas, buscan ser el espejo de sus estudiantes y representar su rol de facilitadores, mejorando permanente y progresivamente sus procesos de enseñanza.

2. En este trabajo se logró determinar que la práctica de la enseñanza muestra distinciones clave entre dos Universidades públicas. La Universidad "A", se caracteriza por su dedicación hacia la Motivación y Autoestima, el Aprendizaje Significativo y la Globalización, ubicando los índices normalizados hacia la derecha de la normal con lo cual se ratifica su enfoque hacia la enseñanza con basada en competencias. Esto permite inferir que el docente relaciona el conocimiento nuevo con el conocimiento asimilado, por una parte, para generar un progreso genuino de aprendizaje por articulación de contenidos, conceptualizaciones y prácticas y, por otra, porque releva el rol del docente como agente activador de las capacidades de sus estudiantes. En tanto que los docentes de la Universidad "B", también focalizados en la Motivación y Autoestima y, Aprendizajes Significativos, ubican las frecuencias de estas categorías al lado opuesto de la curva normal, caracterizando a sus docentes más bien como tradicionales, pero que sin embargo, desde esa posición, validan la categoría de la Globalización como positiva y relevante dentro de su proceso de enseñanza. Es por ello que es posible caracterizar a estos profesores con la doctrina de la enseñanza por objetivos, con cierta proximidad hacia el desarrollo de competencias, permitiendo a los alumnos impulsar sus capacidades para lograr sus metas.

3. En síntesis, en la aplicación del enfoque por competencias, definidas como aquellas que deben desplegar quienes son formados, porque por una parte, éstas son en sí mismas una competencia cuyo constructo exige saber movilizar capacidades, estrategias y destrezas en una totalidad coherente; exige de parte de quienes enseñan un esfuerzo especial de estimulación de la conciencia de trabajo y la reflexión sistemática respecto del que aprende. En primer lugar, colaborando en la descripción, interpretación y búsqueda de conexiones a partir de eventos significativos para el estudiante y en segundo lugar, orientando la actividad docente hacia el desarrollo de competencias que aún quedan pendientes, tales como el pensamiento analítico y comprensivo de la realidad y, así mismo, la reflexión, lógica, capacidad de observación y visión sistémica de una realidad socioeconómica y cultural crecientemente más dinámica y compleja como es la que se observa en el Chile del presente. Esto da pie a nuevas investigaciones, ahora tendientes a focalizar el desarrollo de competencias trasversales, de mayor abstracción, tanto del profesor que enseña como de los estudiantes que las requieren.

\section{REFERENCIAS}

Abela, D. A., Las Técnicas de Análisis de Contenido: Una Revisión Actualizada (en línea) Documentos de Trabajo, Fundación Centro de Estudios Andaluces, Sevilla, Serie Sociología (2002) http://books.google.cl/ Acceso: 20 de mayo (2013) 
Acosta,M. G. y A. Gamino, Modelo curricular del Tecnológico Nacional de México, EISSN: 14094258,Revista Electrónica Educare (Educare ElectronicJournal),20(1), 1-25 (2016)

Argyris, C. y D. Schön, Theory in Practice: Increasing Professional Effectiveness, 1a edición. Jossey-Bass, San Francisco, E.E.U.U (1992)

Barbier, J.M., L'évaluation en formation, 1-295. PUF, París, Francia (1985)

Barbier, J.M., L'analyse des pratiques: questions conceptuelles. EnLánalyse des pratiques professionnelles por O. Fablet y Cl. Blanchard-Laville, pp. 27-49, L'Harmattan, Paris, Francia (1996a)

Barbier, J.M., Savoirs théoriques et savoirs d'action, 1a edición, 1-305. PUF, París, Francia (1996b)

Barbier, J.M., Tutoria y funcion tutorial: Algunas vias de analisis. En Grupos y dispositivos de formaciónpor M. Souto y otros, pp. 129-143, Ediciones novedades educativas, Buenos Aires, Argentina (1999a)

Barbier, J.M, Prácticas de Formación: Evaluación y Análisis, 1a edición, Editorial Novedades Educativas, Buenos Aires, Argentina (1999b)

Barbier, J.M. y O. Galatanu, L'analyse de la singularité des actions: quelques outils conceptuels. En L'analyse de la singularité de l'action por CRF-CNAM, pp. 13-5, PUF, Paris, Francia (2000)

Barbier, J.M., Action et identité: enjeux de la recherche en formation, CRF/Cnam, INRP, Paris, Francia (2001)

Barbier, J.M. y O. Galatanu, Les savoirs d'action: une mise en mots des compétences, 1-325, L'Harmattan Collection Action et Savoir, París, Francia (2004)

Barbier, J.M., E. Bourgeois, G. De Villers e I. Kaddour, Construction des identités et mobilisation des sujets en formation, 301 p, L'Harmattan-Coll. Action et Savoir, París, Francia (2006)

Batanaz, L., El profesor y su implicación en los procesos de cambio en educación, http://www.tendenciaspedagogicas.com/Articulos/1998_e2_10.pdf; ISSN. 1989-8614, Revista Tendencias Pedagógicas, e2, 105-118 (1998)

Bustamante, M., C. Cisternas y P. Villarreal, Evolución de Competencias Profesionales en el Sector de la Salud, Chile, http://www.educandus.cl/ojs/index.php/fcompetencias/article/viewFile/57/56; ISSN 0718-8757, Revista Electrónica de Desarrollo de Competencias (REDEC), 2(4), 105-117 (2009)

Camargo, I.M. y C. Pardo, Competencias Docentes de Profesores de Pregrado: Diseño y Validación de un Instrumento de Evaluación, http://www.redalyc.org/articulo.oa?id=64770211; ISSN: 1657-9267, Univ. Psychol., 7(2), 441-455 (2008)

Carabaña, J., Competencias y universidad, o un desajuste por mutua ignorancia, ISSN: 0210-5934, Bordón 63(1), 15-31 (2011)

Carrera, F. Y J. L. Cioduras, Identificación de las competencias digital del profesor universitario: un estudio exploratorio en el ámbito de las ciencias sociales, ISSN: 1887-4592, Revista de Docencia Universitaria, 10 (2), $273-298(2012)$

Castro, A., Competencias administrativas y académicas en el profesorado de educación media superior, ISSN: 14056666, RMIE, 20(64), 263 - 294 (2015)

Cerrillo M.R. y D. Izuzquiza, Perfil del profesorado universitario,

http://www.redalyc.org/pdf/2170/217017180002.pdf; E-ISSN: 1575-0965, Revista Electrónica

Interuniversitaria de Formación del Profesorado, 8(20), 1-5 (2005)

Checchia, B., Las Competencias del Docente Universitario (en línea) (2009), http://www.fvet.uba.ar/institucional/subir/adjuntos/userfiles/COMPETENCIASDOCENTES.pdf. Acceso: 10 de Marzo (2015)

Coronado, E. Z. y M. R. Estupiñán, Imaginarios sociales sobre el desempeño del docente universitario, http://www.usta.edu.co/otraspaginas/diversitas/doc_pdf/diversitas_12/vol.8no.1/5_imaginarios_sociales.pdf; ISSN: 1794-9998, Divers.: Perspect. Psicol., 8(1), 73-84 (2012) 
Crisol, E., Reseña de "Cómo hicieron los sistemas educativos con mejor desempeño del mundo para alcanzar sus objetivos" Barber, Michael y Mourshed, Mona (2008),

http://www.redalyc.org/pdf/567/56717074023.pdf; ISSN: 1138-414X, Revista de Currículum y Formación de Profesorado, 14(2), 301-303 (2010)

De Miguel, M., Modalidades de Enseñanza centradas en el desarrollo de Competencias: Orientaciones para promover el cambio metodológico en el Espacio Europeo de Educación Superior (en línea), 1aㅡ edición, Oviedo, España, (2005) http://www.uvic.es/sites/default/files/Ensenanza_para_competencias.PDF; Acceso: 10 de marzo (2015)

Dewey, J., Substance, power and quality in Locke, In Philosophical review, 35, 22-38. Reprinted in Freedom and experience: essays presented to Horace M. Kallen, Edited by Sidney Hook and Milton R. (1926)

Díaz, C., P. Martínez, I. Roa y M.G. Sanhueza, Los docentes en la sociedad actual sus creencias y cogniciones pedagógicas respecto al proceso didáctico, doi: 10.4067/S0718-65682010000100025, Polis, 9(25), 421-436 (2010)

Fernández, F., El análisis de contenido como ayuda metodológica para la investigación, Ciencias Sociales Revista de la Universidad de Costa Rica, 2(96), 35-54 (2002)

García, B., J. Loredo y G. Carranza, Análisis de la práctica de los docentes: pensamiento, interacción y reflexión, Revista Electrónica de Investigación Educativa, Especial, 1-15 (2008)

Greca, I.M. y M.A. Moreira, The kinds of mental representations -models, propositions and images -used by college physics students regarding the concept of field. Int J Sci Educ, 19(6), 711-724 (1997)

Johnson-Laird, P., Mental models. En Foundations of cognitive science por M. Posner (ed.), pp. 469-499, MIT Press, Cambridge, Massachusetts, E.E.U.U. (1990)

Labra, P., M. E. Kokaly, C.Iturra, A.Concha, P.Sasso, M. I. Vergara, El enfoque ABP en la formación inicial docente de la Universidad de Atacama: el impacto en el quehacer docente, ISSN: 0716-050X, Revista Estudios Pedagógicos, XXXVII(1), 167-185 (2011)

Legault, A., ¿Una enseñanza universitaria basada en competencias? ¿Por qué?¿Cómo?, Revista electrónica de desarrollo de competencias (REDEC), 5(1) (2012).

LOE; Ley Orgánica Española, Decreto 142/2007, 21822-21870, Barcelona, España (2007)

López, F., El análisis de contenido como método de investigación, http://rabida.uhu.es/dspace/bitstream/handle/10272/1912/b15150434.pdf?sequence=1; ISSN-e: 1575-0345, XXI Revista de Educación, 4, 167-179 (2002)

Marín-García, J.A. y Conchado-Peiró, A., Influencia de los Perfiles Docentes en el Uso y Preferencia de Dinámicas de Trabajo en Grupo con Alumnos Universitarios, doi: 10.4067/S0718-50062012000500002, Form. Univ., 5(5), 3-14 (2012)

Moreno, T., El currículo por competencias en la universidad: más ruido que nueces, ISSN: 0185-2760, Revista de la Educación Superior, XXXIX(154), 77-90 (2010)

Moreno, M.P., M. Nava y M. Campos, Los Comentarios Abiertos como Referente de Evaluación de la Docencia Universitaria: La Conveniencia de su Interpretación y Tratamiento, doi: 10.4067/S071850062014000100006, Form. Univ., 7(1), 41-48 (2014)

Organización para la Cooperación y el Desarrollo Económico: OCDE, Revisión de políticas nacionales de educación (2004), (en línea), París y Ministerio de Educación, Chile, http://www.oecdilibrary.org/docserver/download/1404094e. pdf?expires=1430336185\&id=id\&accname=oid035281\&checksum =7869744C677FE9C234FCCE105C4A860D; Acceso 10 de Marzo (2015)

Ortiz, M. y E. Cires, Diseño curricular por competencias. Aplicación al macrocurrículo, ISSN: 20772874,http://www.iisue.unam.mx/seccion/perfiles/; Edumecentro, 4(1), 10-17 (2012)

Palominos, F., M. Méndez, y R. Barrera, Sistema de Perfeccionamiento Orientado a Competencias para Docentes de la Educación Superior, doi: 10.4067/S0718-50062014000300003, Form. Univ., 7(3), 1-22 (2014) 
Patiño, L. y M. Rojas, Subjetividad y Subjetivación de las Prácticas Pedagógicas en la Universidad. Educ. Educ., 12(1), 93-105 (2009)

Pavié, A., Formación docente: hacia una definición del concepto de competencia profesional docente, REIFOR, 14(1), 67-80(2011) (en línea)

http://www.aufop.com/aufop/uploaded_files/articulos/1301587967.pdf; Acceso 18 de marzo (2016)

Perrenoud, P., La Formación de los Docentes en el Siglo XXI (en línea)

http://www.unige.ch/fapse/SSE/teachers/perrenoud/php_main/php_2001/2001_36.html; (2001) Acceso: 10 de Marzo (2013)

Popper, K.R., En busca de un mundo mejor, 1-320, Editorial PAIDOS, España (1994)

Rodríguez, G., La evaluación de la actividad docente en la Universidad: entre el sueño y la realidad, Revista de Investigación Educativa, 18(2), 417-432 (2000)

Rué, J., Formar en competencias en la universidad: entre la relevanciay la banalidad, Red U. Revista de Docencia Universitaria, Num. Monograf. 1ํ, 1-19(2008)

http://tecnologiaedu.us.es/mec2011/htm/mas/3/31/64.pdf; Acceso : 18 de marzo (2016)

Spencer, L., Competence at Work, Models for Superior Performance, $1^{\text {a }}$ edición, 384 p., Editorial John Wiley \& Sons, Inc., Nueva York, E.E.U.U. (1993)

Vadillo, G. y C. Klingler., Teoría y práctica de éxito en Latinoamérica y España, Editorial Mc Graw Hill/ Interamericana de México, México (2004) 
